\title{
PREVALÊNCIA DO HBsAg EM GESTANTES DE PASSO FUNDO, RS: estudo comparativo entre os sistemas de saúde público e privado
}

\author{
Andressa Pilonetto LIELL'1, Daiane WEBER ${ }^{1}$, Camila TOSCAN ${ }^{1}$, \\ Fernando FORNARI ${ }^{2}$ e Luiz Fernando MADALOSSO ${ }^{3}$
}

\begin{abstract}
RESUMO - Estimou-se a prevalência do HBsAg em gestantes dos sistemas de saúde público e privado de Passo Fundo, RS, Brasil. Foram analisados prontuários de 3.573 gestantes de 6 serviços públicos e 16 privados. O nível socioeconômico foi estimado comparando-se o número de gestações. A prevalência geral de HBsAg foi de $0,7 \%$, não diferindo entre os sistemas público e privado $(0,64 \%$ vs $0,79 \% ; P=$ 0,603). O número de gestações foi maior nas gestantes do sistema público [2 (1-6) vs $1(1-3) ; P=0,0001]$. Conclui-se que a prevalência do HBsAg em gestantes de Passo Fundo é baixa e independente do nível socioeconômico.
\end{abstract}

DESCRITORES - Antígeno de superfície da hepatite B. Hepatite B. Gravidez. Passo Fundo, RS., Brasil.

\section{INTRODUÇÃO}

A hepatite B é uma doença infecciosa causada pelo vírus B. Representa um problema de saúde pública mundial, podendo ser prevenida com vacinação. Segundo estimativas da Organização Mundial de Saúde, mais de 2 bilhões de indivíduos encontram-se infectados pelo vírus da hepatite B (VHB), dos quais 350 milhões apresentam infecção crônica $^{(5)}$.

Os principais grupos de risco para a infecção pelo VHB incluem recém-nascidos de mães infectadas, usuários de drogas ilícitas, pessoas com múltiplos parceiros sexuais, homens que fazem sexo com homens, hemodialisados, profissionais da saúde e indivíduos pertencentes às classes socioeconômicas menos favorecidas ${ }^{(2,3)}$. A presença do antígeno de superfície do VHB (HBsAg) no sangue de gestantes é o marcador utilizado para rastreamento e prevenção da contaminação de recém-nascidos, o que caracteriza a transmissão vertical do VHB.

A transmissão vertical por sua vez é considerada a principal forma de contágio da doença e costuma ser acompanhada de altas taxas de cronicidade. A contaminação de recém-nascidos pelo VHB acarreta elevado risco de desenvolvimento de cirrose e carcinoma hepatocelular, que podem ocorrer em adultos jovens ${ }^{(5)}$. O rastreamento do HBsAg em gestantes possibilita a tomada de medidas para prevenir a contaminação do recém-nascido.
Este estudo foi realizado com o objetivo de estimar a prevalência do HBsAg em gestantes de Passo Fundo, RS, oferecendo suporte para a implementação de medidas profiláticas.

\section{MÉTODOS}

Realizou-se estudo retrospectivo com análise de prontuários de gestantes dos sistemas de saúde público e privado do município de Passo Fundo, RS, Brasil, atendidas entre janeiro de 2003 e dezembro de 2005 . Foram incluídas pacientes cujos prontuários continham informações sobre a pesquisa do HBsAg sérico durante a gestação. De uma amostra representativa de gestantes dos sistemas público e privado, pesquisaram-se os números de gestações com a finalidade de se comparar o nível socioeconômico das pacientes dos dois sistemas de saúde. O protocolo do estudo foi aprovado pela Comissão de Ética em Pesquisa da Universidade de Passo Fundo.

Os dados dos pacientes do sistema público foram pesquisados em todos os seis Centros de Atenção Integral à Saúde (CAIS) do município, locais de atendimento público, onde se realizam consultas obstétricas e que estão localizados nas diversas regiões, abrangendo toda a população da cidade. Tais centros foram visitados com autorização do Secretário da Saúde vigente do município e dos responsáveis de cada Unidade de Saúde.

Instituição: Universidade de Passo Fundo, RS

${ }^{1}$ Curso de Medicina da Faculdade de Medicina, Universidade de Passo Fundo; ${ }^{2}$ Residência Médica em Gastroenterologia do Hospital São Vicente de Paulo, Passo Fundo; ${ }^{3}$ Departamento de Gastroenterologia da Faculdade de Medicina, Universidade de Passo Fundo.

Correspondência: Prof. Luiz Fernando Madalosso - Rua Uruguai, 1555 - 1ํandar - Passo Fundo, RS. E-mail: Ifmadalosso@brturbo.com.br 
Para compor o grupo de gestantes do sistema de saúde privado, foram incluídas 16 clínicas particulares, de acordo com os seguintes critérios: 1. atendimento particular ou por convênios; 2. localização nas áreas centrais do município; 3. consentimento para fornecer os dados solicitados. Os dados da rede privada foram coletados de acordo com informações fornecidas pelo responsável de cada clínica.

O número de gestações foi apresentado como mediana (amplitude) e comparado com teste $t$ não-paramétrico (MannWhitney). A prevalência de HBsAg em cada grupo de gestantes foi apresentada em percentual e comparada através de teste do Qui ao quadrado. O valor de $P$ foi considerado significativo se inferior a $5 \%$.

\section{RESULTADOS}

O total de 4.497 prontuários foi avaliado, sendo 3.103 (69\%) do sistema público e 1.394 (31\%) do privado. Foram excluídos 924 prontuários do sistema público (30\%) por não conterem o resultado do HBsAg, e nenhum do privado, perfazendo uma amostra final de 3.573 prontuários (proporção público:privado $=6: 4$ ).

Esses dados estão apresentados na Tabela 1. A prevalência geral de HBsAg foi $0,7 \%$, não se observando diferença estatisticamente significante entre a prevalência do $\mathrm{HBsAg}$ em gestantes dos sistemas de saúde público $(0,64 \%)$ e privado $(0,79 \%)$. O número de gestações foi significantemente maior na amostra de pacientes do sistema público em relação ao do sistema privado, indicando menor nível socioeconômico nas gestantes do sistema público.

TABELA 1. Prevalência do HBsAg em gestantes do sistemas público e privado de Passo Fundo

\begin{tabular}{lccc} 
& $\begin{array}{c}\text { Sistema público } \\
(\mathrm{n}=2179)\end{array}$ & $\begin{array}{c}\text { Sistema privado } \\
(\mathrm{n}=1394)\end{array}$ & $\mathbf{P}$ \\
\hline $\begin{array}{l}\text { HBsAg positivo, } \mathrm{n}^{\circ}(\%) \\
\mathrm{N}^{\circ} \text { de gestações* }\end{array}$ & $14(0,64)$ & $11(0,79)$ & 0,603 \\
Mediana (amplitude) & $2(1-6)$ & $1(1-3)$ & 0,0001 \\
\hline
\end{tabular}

\section{DISCUSSÃO}

A hepatite B é uma doença de considerável impacto em saúde pública. Estratégias de prevenção são fundamentais para seu efetivo controle, incluindo o uso judicioso de vacinas e de imunoglobulina específica. Para tal, é necessário o conhecimento de suas taxas de prevalência, especialmente em populações de risco, como gestantes.

Baseado nestes princípios, o objetivo deste estudo foi estimar a prevalência do HBsAg em gestantes atendidas em um pólo de atenção à saúde, comparando-se taxas de prevalência entre os sistemas público e privado.

A prevalência geral do HBsAg foi $0,7 \%$, configurando a região estudada como área de baixa prevalência $(<2 \%)$ segundo o "Centers for Diseases Control and Prevention" (CDC). É importante ressaltar o grande número de pacientes incluído (mais de 3.500) e a adequada representatividade dos centros de saúde pesquisados, abrangendo serviços públicos e privados de uma cidade de médio porte, com elevada circulação de pacientes. Em concordância com esse achado, estudos realizados em outras regiões populosas do Brasil têm descrito prevalência de $\mathrm{HBsAg}$ entre 0,4 e $0,8 \%{ }^{(1,4)}$.

Considerando-se que a transmissão da hepatite B possa estar aumentada em populações desfavorecidas socioeconomicamente ${ }^{(3)}$, esperar-se-ia maior prevalência do HBsAg nas gestantes do sistema público em detrimento do privado. No entanto, as taxas de HBsAg foram similares nessas populações, questionando-se, assim, a desigualdade socioeconômica entre as pacientes dos sistemas público e privado. Teve-se o cuidado de estimar e comparar o nível socioeconômico das gestantes atendidas nesses sistemas, levantando-se o número de gestações por paciente em amostras aleatórias das referidas populações, considerando-se que maior densidade familiar seja indicativa de menor nível socioeconômico. Observou-se número significativamente maior de nascidos vivos das pacientes do sistema público, indicando que a prevalência de HBsAg independe do nível socioeconômico neste estudo. Possíveis explicações para a reduzida taxa de prevalência de HBsAg incluem a melhora da qualidade dos serviços de saúde pública, com a vacinação em massa, a crescente utilização de medidas preventivas contra doenças sexualmente transmissíveis e a melhoria dos índices de nutrição em populações menos favorecidas.

Em resumo, estimou-se a prevalência do HBsAg em gestantes atendidas nos sistemas público e privado de um grande centro de atenção à saúde. $\mathrm{O}$ principal achado foi a reduzida taxa de prevalência deste marcador, independente do nível socioeconômico da população estudada. Melhor aprimoramento das medidas de saúde pública poderia ser capaz de erradicar a hepatite B na região estudada.

Liell AP, Weber D, Toscan C, Fornari F, Madalosso LF. Prevalence of HBsAg in pregnant women of Passo Fundo, RS, Brazil: comparative study between public and private health systems. Arq Gastroenterol. 2009;46(1): 89-91.

ABSTRACT - We estimated the prevalence of HBsAg in pregnant women assisted in public and private health systems of the city of Passo Fundo, RS, Brazil. Health registers of 3,573 patients assisted in 6 public and 16 private services were analyzed. The socioeconomic level was estimated comparing the number of pregnancies. Overall prevalence of HBsAg was $0.7 \%$, being similar comparing public and private systems $(0.64$ vs $0.79 \% ; P=0.603)$. Pregnancy number was higher in patients of the private system [2 (1-6) vs $1(1-3) ; P=0.0001]$. The prevalence of HBsAg in pregnant women of Passo Fundo was low and independent of the socioeconomic level.

HEADINGS - Hepatitis B surface antigens. Hepatitis B. Pregnancy. Passo Fundo, RS, Brazil. 


\section{REFERÊNCIAS}

1. Arraes LCA, Sampaio S, Barreto S, Guilherme MAS, Lorenzato F. Prevalência de hepatite B em parturientes e perfil sorológico perinatal. Rev Bras Ginecol Obstet. 2003;25:571-5

2. Duarte G, Mussi-Pinhata MM, Martinez R, Lemos C, Figueiredo EML, Quintana SM Frequency of pregnant women with HBsAg in a Brazilian community. Rev Panam Salud Publica. 1997; 1:35-40

3. Miranda AE, Alves MC, Neto RL, Areal KR, Gerbase AC. Seroprevalence of HIV, hepatitis B virus, and syphilis in women at their first visit to public antenatal clinics in Vitória, Brazil. Sex Transm Dis. 2001;28:710-3.
4. Reiche EMV, Morimoto HK, Farias GN, Hisatsugu KR, Geller L, Gomes ACLF, Inoue HY, Rodrigues G, Matsuo T. Prevalência de tripanossomíase americana, sífilis, toxoplasmose, rubéola, hepatite $\mathrm{B}$, hepatite $\mathrm{C}$ e da infecção pelo vírus da imunodeficiência humana, avaliada por intermédio de testes sorológicos, em gestantes atendidas no período de 1996 a 1998 no Hospital Universitário Regional Norte do Paraná (Universidade Estadual de Londrina, Paraná, Brasil). Rev Soc Bras Med Trop. 2000;33:519-27.

5. WHO. World Health Organization. Epidemic and pandemic alert and response (EPR), 2002. [Online] Julho 2006 [citado em 11 de julho de 2006] Disponível: $<$ http://www. who.int/csr/disease/hepatitis>.

Recebido em 20/3/2008. Aprovado em 6/5/2008 